\title{
Corticosteroids and Fetal Intervention Interact to Alter Lung Maturation in Preterm Lambs
}

\author{
BANNIE L. TABOR, JAMES F. LEWIS, MACHIKO IKEGAMI. \\ DANIEL POLK, AND ALAN H. JOBE \\ Departments of Obstetrics/Gynecology /B.L.T.] and Pediatrics [J.F.L., M.I., D.P., A.H.J.]. \\ Harbor-University of California, Los Angeles Medical Center, Torrance, California 90502
}

\begin{abstract}
The relationship between cortisol infusion and time of fetal catheterization on postnatal lung function of prematurely delivered lambs was investigated with the hypothesis that the intervention of catheterization would alter fetal responsiveness to the maturational effects of corticosteroids. Fetal catheterization was performed on d 117 or on d 122 of gestation. Cortisol or saline control infusions were begun on d 126, with delivery $60 \mathrm{~h}$ later on d 128. The animals were ventilated for $1.25 \mathrm{~h}$ after delivery, and compliance, the ventilation efficiency index, labeled albumin leak into and out of the lungs, alveolar and lung saturated phosphatidylcholine and surfactant protein $\mathbf{A}$ were measured to evaluate lung performance and biochemical indicators of maturation. Cortisol improved compliance and ventilation efficiency and decreased labeled albumin recovery without changing alveolar saturated phosphatidylcholine or surfactant protein $A$ in the animals catheterized at 122 d relative to 122-d saline-infused animals. However, the animals catheterized at $117 \mathrm{~d}$ and infused with saline were as mature as assessed by compliance and ventilation efficiency as the 122-d cortisol-treated animals. The 117-d cortisol-infused animals had significantly augmented lung function relative to either 117-d saline-infused or 122-d cortisol-treated lambs and were the only group that had increased alveolar surfactant protein $A$ and lung saturated phosphatidylcholine pool sizes. This study demonstrates that the response of the fetal lung to a maturational agent such as cortisol is dependent on the history of previous fetal interventions. (Pediatr Res 35: 479-483, 1994)
\end{abstract}

\section{Abbreviations}

Sat PC, saturated phosphatidylcholine SP-A, surfactant protein A

After Liggins' observation in 1969 (1) that cortisol infusions to fetal sheep induced preterm labor and resulted in unanticipated lung maturation, many reports demonstrated that maternal corticosteroids decrease the incidence and severity of neonatal respiratory distress syndrome (2). The clinical reduction in respiratory distress syndrome and infant mortality likely represents effects of corticosteroids on the preterm fetal lung as well as on other organ systems (3). Although surfactant pools can increase after prenatal corticosteroid treatment in selected experimental animal models (2), corticosteroids improve lung compliance,

Received July 26, 1993: accepted November 19, 1993.

Correspondence and reprint requests: Alan Jobe, M.D., Ph.D., Department of Pediatrics, 1124 W. Carson St., RB-1, Torrance, CA 90502.

Supported by Grant HD-20618 from the National Institutes of Health. increase lung volumes, and decrease epithelial and endothelial permeability without increasing alveolar surfactant pool sizes in ventilated preterm rabbits and sheep $(4,5)$. Prenatal corticosteroid exposure also can alter neuroendocrine adaptation and increase blood pressure and cardiac performance in the preterm resulting indirectly in improvements in pulmonary performance (6). Despite these many observations, inconsistent responses to prenatal corticosteroid exposure continue to be reported. For example, Liggins' group found a modest increase in pressurevolume curves of preterm lambs in one study but no effect in a second study $(7,8)$. These disparate results may reflect the difficulty of manipulating a fetus without inadvertently influencing lung maturation (9). In fetal sheep, the manipulation is generally the placement of fetal catheters for delivery of agents to be tested for maturational responses. Moreover, prenatal exposure to corticosteroids is efficacious in only about $50 \%$ of human fetuses at risk for preterm delivery (3). We thought that a fetus may need to be primed by prior adverse pregnancy events to consistently respond to corticosteroids. Our hypothesis was that the timing of surgery to place the fetal infusion catheter would alter the fetal milieu and thus alter the fetal response.

\section{MATERIALS AND METHODS}

Experimental design. Pregnant Western mixed-breed ewes with twin pregnancies were divided into two groups based on the time of surgery. Fetal catheterization was performed in the early surgery group at $117 \pm 1 \mathrm{~d}$ gestational age and at $122 \pm 1 \mathrm{~d}$ gestational age in the late surgery group. Cortisol infusions or the control saline infusions were begun on $\mathrm{d} 126$, with delivery $60 \mathrm{~h}$ later on $\mathrm{d} 128$. The animals catheterized at $122 \mathrm{~d}$ were reported in a concurrent investigation of combination hormonal and surfactant therapy on preterm lung function (4). The gestational age of $128 \mathrm{~d}$ was selected to correspond with that used by Liggins' group $(7,8)$ and by other investigators studying fetal lung maturation (10).

Fetal catheterization. Guidelines for the care and use of animals approved by our institutional review board were followed during the experiment. On the day of surgery, each ewe was anesthetized with $1.2 \mathrm{mg}$ of atropine and $700 \mathrm{mg}$ of ketamine given by intramuscular injection, followed by a continuous intravascular infusion of ketamine at $100 \mathrm{mg} / \mathrm{h} \mathrm{(4).} \mathrm{Through} \mathrm{a}$ midline abdominal incision, a single fetal hind limb was exposed via a small hysterotomy. With use of local field block with lidocaine, the dorsal hind limb artery and vein were catheterized with polyethylene tubing to the level of the abdominal aorta and inferior vena cava, respectively. The fetal wound and hysterotomy were then closed and the procedure repeated on the second twin. Before closing the abdominal incision, the catheters were exteriorized to the maternal flank. The catheters were then filled with $1000 \mathrm{U} / \mathrm{mL}$ heparin, capped, and secured in a cloth pouch on the ewe's flank. The catheters were flushed daily to maintain 
patency. The ewe received $80 \mathrm{mg}$ of gentamicin and $500 \mathrm{mg}$ of oxacillin preoperatively and twice daily for $1 \mathrm{~d}$ after surgery.

Cortisol infusions. Each ewe in the 117- and 122-d surgery groups was randomized to either saline infusion or cortisol infusion. One lamb of each twin previously catheterized at 122 $\mathrm{d}$ was used for this protocol. The second 122-d fetus received the same fetal treatment but was treated postnatally with surfactant for another protocol (4). Both fetuses catheterized at $117 \mathrm{~d}$ were studied for this protocol. This resulted in four treatment groups: 117-d surgery/saline infused, 117-d surgery/cortisol infused, 122$\mathrm{d}$ surgery/saline infused, and 122-d surgery/cortisol infused. Treatment was begun for all fetuses on $d 126$ of gestation. The corticosteroid-treated animals received a continuous infusion of $0.75 \mathrm{mg}$ of cortisol $/ \mathrm{kg}$ estimated body weight $/ \mathrm{h}$ or $2.1 \mu \mathrm{mol} / \mathrm{kg} /$ $\mathrm{h}$ as Solu-Cortef (Upjohn, Kalamazoo, MI). This dose achieves stable cortisol levels similar to those reported for normal term sheep before spontaneous delivery and is similar to the dose used by others $(7,11)$. The cortisol was diluted in heparinized saline (1 $\mathrm{U} / \mathrm{mL}$ ) so that $2 \mathrm{~mL} / \mathrm{h}$ were infused. The saline-infused animals received a continuous infusion of heparinized saline at the same rate. The infusions were given via the fetal catheters using battery-operated peristaltic infusion pumps (Travenol Laboratories, NH), which were placed in cloth pouches on the flank of each ewe.

Postnatal studies. After the 60 -h infusion period at $128 \mathrm{~d}$ gestational age, each ewe was anesthetized with $1 \mathrm{~g}$ of ketamine and $2.4 \mathrm{mg}$ of atropine given by intramuscular injection followed by a continuous infusion of ketamine at $100 \mathrm{mg} / \mathrm{h}$. After lidocaine infiltration of the tissue over the maternal trachea, a tracheostomy was performed to protect the maternal airway and allow for mechanical ventilation. Maternal blood gases and $\mathrm{pH}$ were monitored during fetal delivery via cesarean section through a midline abdominal and uterine incision. After delivery of the head and shoulders, each lamb received $10 \mathrm{mg} / \mathrm{kg}$ ketamine and $0.1 \mathrm{mg} / \mathrm{kg}$ acepromazine by intramuscular injection. After administration of $2 \%$ lidocaine to the skin and subcutaneous tissues over the trachea, a $4.5-\mathrm{mm}$ inner diameter endotracheal tube was tied into the trachea via a tracheostomy and $5-10 \mathrm{~mL}$ of fetal lung fluid were aspirated. Radiolabeled ${ }^{125} \mathrm{I}$-albumin $(5 \mu \mathrm{Ci})$ was added to the aspirated fetal lung fluid, which was then returned to the lungs via the endotracheal tube. The lamb was delivered, superficially dried, weighed, and transferred to a radiant warming bed for ventilation with a pressure-limited infant ventilator. The initial ventilatory settings were as follows: rate, 40 breaths/min; inspiratory time, $0.7 \mathrm{~s}$; positive end-expiratory pressure, $3 \mathrm{~cm} \mathrm{H}_{2} \mathrm{O}$; and fraction of inspired oxygen, $100 \%$. Immediately after delivery and the start of ventilation, a $5 \mathrm{Fr}$ catheter was passed via an umbilical artery into the distal aorta and subsequently infused with $5 \%$ dextrose at $4 \mathrm{~mL} / \mathrm{kg} / \mathrm{h}$. Ventilation was regulated by adjusting only in the peak inspiratory pressure to try to maintain $\mathrm{PCO}_{2}$ values between 4 and $6 \mathrm{kPa}$ (30-45 $\mathrm{mm} \mathrm{Hg}$ ). A maximum peak inspiratory pressure of 40 $\mathrm{cm} \mathrm{H}_{2} \mathrm{O}$ was used to avoid pneumothoraces (4). Arterial blood gases were measured every $15 \mathrm{~min}$ to guide the ventilatory adjustments. After 15 min of ventilation, each lamb received 10 $\mu \mathrm{Ci}{ }^{131} \mathrm{I}$-albumin via the umbilical artery catheter. Radiant heaters and heating lamps were used to maintain core body temperature. Tidal volumes were measured with a pneumotachometer (12). Just before the end of the 1.25 -h period of ventilation, a sample of blood was collected in a heparinized syringe for subsequent $\mathrm{Hb}$ and radioisotope measurements. Each lamb was then given $30 \mathrm{mg} / \mathrm{kg}$ pentobarbital by intravascular injection to induce deep anesthesia and the endotracheal tube was clamped. After $5 \mathrm{~min}$ to allow for resorption atelectasis, the chest was opened and the heart removed.

Processing lungs and samples. The lungs were carefully removed from the chest and weighed, and an alveolar wash was performed as previously reported using iced saline to fully distend the lungs (13). The saline was withdrawn with a syringe and the procedure was repeated five times. The alveolar washes were pooled and the total volume was recorded. The lungs were then homogenized in $400 \mathrm{~mL}$ of water and the lung homogenate volume recorded. Aliquots of the alveolar wash, lung homogenate, and blood, along with triplicate samples of ${ }^{131} \mathrm{I}$ - and ${ }^{125} \mathrm{I}$ albumin used for the intravascular and intratracheal injections were assayed for radioactivity. The contribution of intravascular blood to the radioactivity associated with the lung tissue was calculated using the $\mathrm{Hb}$ content of both blood and lung tissue. The blood contribution was subsequently subtracted from the total radioactivity of the lung (12). Chloroform:methanol (2:1, $\mathrm{vol} / \mathrm{vol}$ ) extracts of aliquots of alveolar wash and lung homogenate were treated with osmium tetroxide and Sat PC was isolated by alumina column chromatography and quantified by phosphorous assay (14-16). SP-A in alveolar washes was measured using a previously described RIA (4).

Material. Radiolabeled albumin was prepared by iodination with ${ }^{131}$ I or ${ }^{125}$ I using chloramine-T and monomer standard BSA (12). The iodinated albumin was extensively dialyzed, and the incorporation was verified by trichloroacetic acid precipitation.

Data analysis. The ${ }^{125} \mathrm{I}$ - and ${ }^{131} \mathrm{I}$-albumin radioactivities of alveolar washes and the lung tissues were expressed as percent recovery of the quantities given to the animals. Compliance values were calculated by dividing the tidal volume $(\mathrm{mL})$ by the peak inspiratory pressure $\left(\mathrm{cm} \mathrm{H}_{2} \mathrm{O}\right)$ and the body weight $(\mathrm{kg})$. A ventilatory efficiency index (VEI) was calculated for each animal as a measure of ventilation and gas exchange that includes ventilatory pressures, rate, and $\mathrm{PCO}_{2}$ (17): $\mathrm{VEI}=3800 /(\mathrm{P} \times \mathrm{F} \times$ $\mathrm{PCO}_{2}$ ), where 3800 is a constant relating to $\mathrm{CO}_{2}$ production, $P$ is the peak inspiratory pressure minus positive end-expiratory pressure, $\mathrm{F}$ is ventilatory frequency, and $\mathrm{PCO}_{2}$ is arterial carbon dioxide pressure in $\mathrm{mm} \mathrm{Hg}$. All values are reported as means \pm SEM. Statistical analysis was performed by using analysis of variance followed by Student-Newman-Keuls multiple comparison procedure. Significance was accepted at $p<0.05$.

\section{RESULTS}

Description of animals. There were no differences in body weights, lung weights, or lung/body weight ratios among the four groups (Table 1). The fetal weight was overestimated by about $40 \%$. As a result, the corticosteroid-treated animals received approximately $1 \mathrm{mg}$ cortisol $/ \mathrm{kg} / \mathrm{h}(2.8 \mu \mathrm{mol} / \mathrm{kg} / \mathrm{h})$. The ventilatory strategy achieved $\mathrm{pH}, \mathrm{PO}_{2}$, and $\mathrm{PCO}_{2}$ values that were within normal limits for all groups except the saline control group catheterized at $122 \mathrm{~d}$ (Table 1). The $\mathrm{PCO}_{2}$ values of the 122-d saline group were higher than for all other groups and could not be successfully lowered despite the use of a mean peak inspiratory pressure of $40 \mathrm{~cm} \mathrm{H}_{2} \mathrm{O}(p<0.01)$. In contrast, the cortisol-treated animals catheterized at $117 \mathrm{~d}$ had $\mathrm{PCO}_{2}$ values in the low normal range despite the use of much lower ventilatory pressures than for the other three groups $(p<0.01)$. These ventilatory pressure requirements were also reflected in the calculated compliances (Fig. 1). Compliance values for the 122-d control animals were significantly lower $(p<0.05)$ than those observed in the 122-d cortisol and 117-d control groups, which were comparable. The 117-d cortisol group had a mean compliance value that was at least twice that of any other group $(p<$ 0.01 ). The 117-d cortisol group also had a greater than 2-fold improvement in the ventilation efficiency index in comparison with the other groups $(p<0.01)$, indicating more efficient ventilation.

Labeled albumin recovery. The recovery of ${ }^{131} \mathrm{I}$-albumin given by intravascular injection in alveolar washes and total lung (the sum of alveolar wash plus lung tissue-associated ${ }^{131} \mathrm{I}$-albumin) is a measure of the interval accumulation of intravascular protein into the lungs over $1 \mathrm{~h}$ (Fig. 2). A similar amount of labeled albumin accumulated in the lungs of the 117-d and 122-d control animals, whereas less than $50 \%$ of this amount was found in the lungs of cortisol-treated animals. These differences were significant for the 122-d control versus either cortisol-treated group ( $p$ 
Table 1. Description of animals

\begin{tabular}{|c|c|c|c|c|}
\hline & \multicolumn{2}{|c|}{ Catheterized at $117 \mathrm{~d}$} & \multicolumn{2}{|c|}{ Catheterized at $122 \mathrm{~d}$} \\
\hline & Saline & Cortisol & Saline & Cortisol \\
\hline$n$ total ( $n$ male) & $6(3)$ & $6(2)$ & $4(2)$ & $8(5)$ \\
\hline Body weight (kg) & $2.1 \pm 0.1$ & $2.4 \pm 0.1$ & $2.5 \pm 0.2$ & $2.2 \pm 0.1$ \\
\hline Lung weight $(\mathrm{g})$ & $86 \pm 3$ & $83 \pm 4$ & $99 \pm 11$ & $87 \pm 6$ \\
\hline \multicolumn{5}{|l|}{ Values at $1.25 \mathrm{~h}$} \\
\hline $\mathrm{pH}$ & $7.30 \pm 0.03$ & $7.39 \pm 0.04$ & $7.16 \pm 0.05^{*}$ & $7.37 \pm 0.02$ \\
\hline $\mathrm{PO}_{2}(\mathrm{kPa}) \dagger$ & $16.8 \pm 3.7$ & $20 \pm 5.4$ & $13.1 \pm 7.5$ & $22.0 \pm 3.3$ \\
\hline $\mathrm{PCO}_{2}(\mathrm{kPa}) \dagger$ & $5.8 \pm 0.5$ & $4.4 \pm 0.1$ & $10.2 \pm 1.9^{*}$ & $5.6 \pm 0.3$ \\
\hline Peak inspiratory pressure $\left(\mathrm{cm} \mathrm{H}_{2} \mathrm{O}\right)$ & $35.3 \pm 1.5$ & $21.2 \pm 1.7^{*}$ & $39.8 \pm 0.6$ & $36.1 \pm 2.2$ \\
\hline
\end{tabular}

* Different from other groups, $p<0.01$.

$+\mathrm{kPa} \times 7.55=\mathrm{mm} \mathrm{Hg}$.
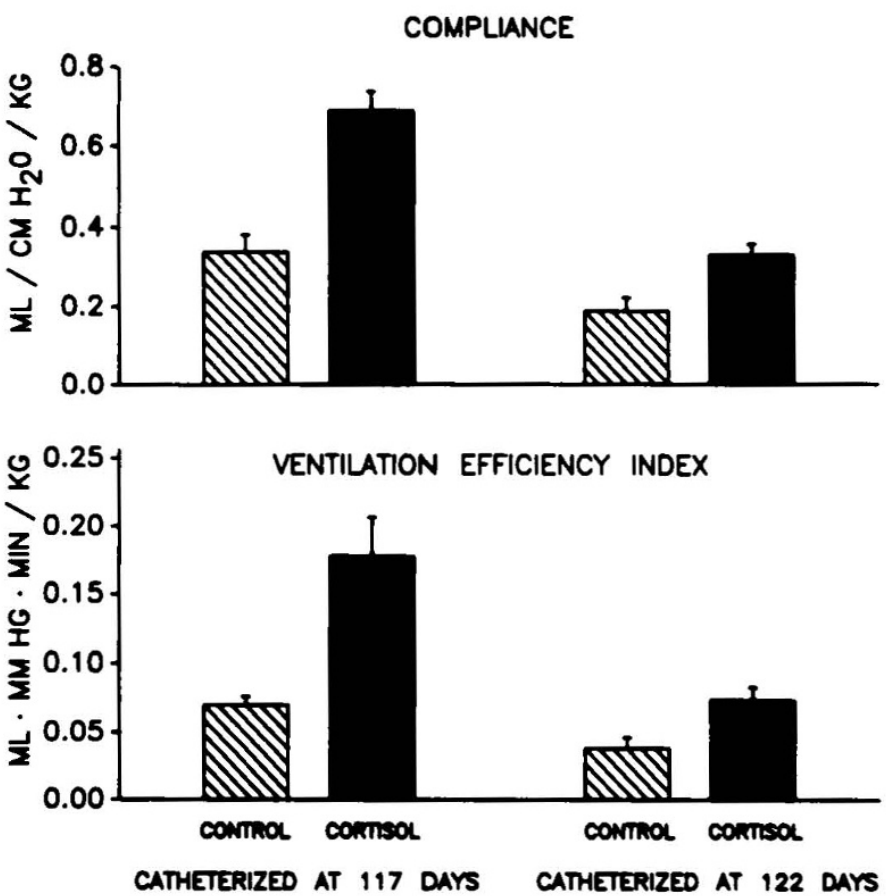

Fig. 1. Effects of time of catheterization and cortisol on lung compliance and ventilation efficiency index measured at $1.25 \mathrm{~h}$. All lambs were infused for $60 \mathrm{~h}$ with saline or cortisol before delivery at $128 \mathrm{~d}$ gestational age. Both compliance and ventilation efficiency index increased significantly in the 117-d cortisol-treated animals relative to all other groups $(p<0.05)$. Cortisol in 122-d animals also increased compliance and ventilation efficiency index relative to those of the 122-d controls $(p<$ 0.05 ). Compliance of control 117-d animals was higher than that for $122-d$ control lambs $(p<0.05)$. The number of animals in each group are given in Table 1 .

$<0.05$ ). Alveolar washes contained $32-38 \%$ of the total labeled albumin in the lungs in all groups. Therefore, the percent recovery of labeled albumin was decreased in the alveolar washes of cortisol-treated animals relative to control animals. There were no differences in recoveries related to the time of catheterization.

The recovery of the ${ }^{125} \mathrm{I}$-albumin mixed with the fetal lung fluid at delivery was used to estimate the movement of protein from the airspaces into the lung tissue and subsequently out of the lungs (Fig. 3). For this measurement, a higher alveolar wash recovery indicates a more intact epithelial barrier. The 117-d control group had almost twice the alveolar recovery noted for the $122-\mathrm{d}$ control group $(p<0.05)$. Cortisol treatment had little effect on alveolar recovery in the 122-d animals, but alveolar recoveries increased nearly 2 -fold with cortisol infusion in the $117-\mathrm{d}$ group $(p<0.01)$. All groups were significantly different from each other for the measurement of labeled albumin recovery in the total lung (alveolar wash plus lung tissue). The early
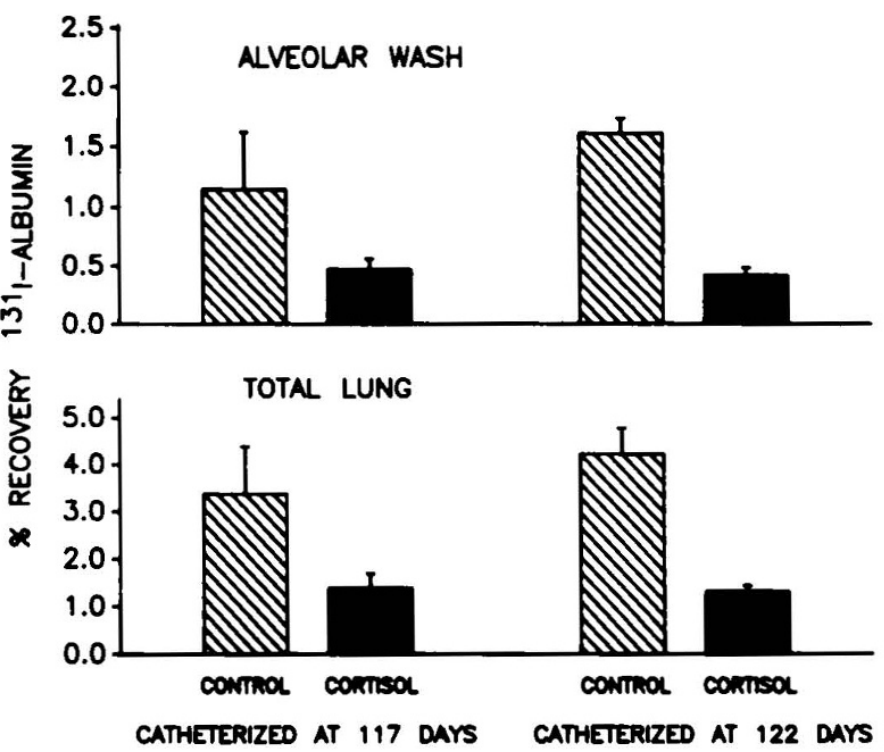

Fig. 2. Recovery of intravascular ${ }^{131} \mathrm{I}$-albumin in alveolar washes and total lung (alveolar wash plus lung tissue) $1 \mathrm{~h}$ after intravascular injection. Recoveries were lower in both cortisol-treated groups than in the 122-d control group in both the alveolar wash and lung tissue $(p<0.05)$. Values for the 117-d controls were similar to those of the 122-d controls. Animal numbers for each group are given in Table 1 .

catheterized groups had recoveries greater than $75 \%$, and cortisol treatments increased recovery at both times of catheterization ( $p$ $<0.05$ ).

Sat PC and SP-A pool sizes. The amount of Sat PC recovered in the alveolar wash was used to estimate the size of the surfactant pool in the airspaces. There was an increase in the amount of Sat PC recovered in the alveolar wash of 117-d cortisol animals compared with the other groups, although the difference was not statistically significant by analysis of variance (Fig. 4). The amount of Sat PC in the total lung in the 117-d cortisol animals was almost twice that of the other groups $(p<0.01)$, which were comparable. The amount of alveolar wash SP-A in the 117-d cortisol animals was twice that of the 117-d controls $(p<0.05)$ and more than 6 times that of the 122-d animals $(p<0.01)$. Because there also was an increase in the amount of alveolar wash Sat PC, the ratio of SP-A to Sat PC was calculated. There were no differences between the two 117-d groups. The ratio in both of these groups was approximately twice that of the 122-d groups, but the differences were not significant.

\section{DISCUSSION}

Preterm lambs at $128 \mathrm{~d}$ gestation have a severe degree of surfactant deficiency and lung immaturity (18). Antenatal corticosteroids have not consistently enhanced lung maturation given 


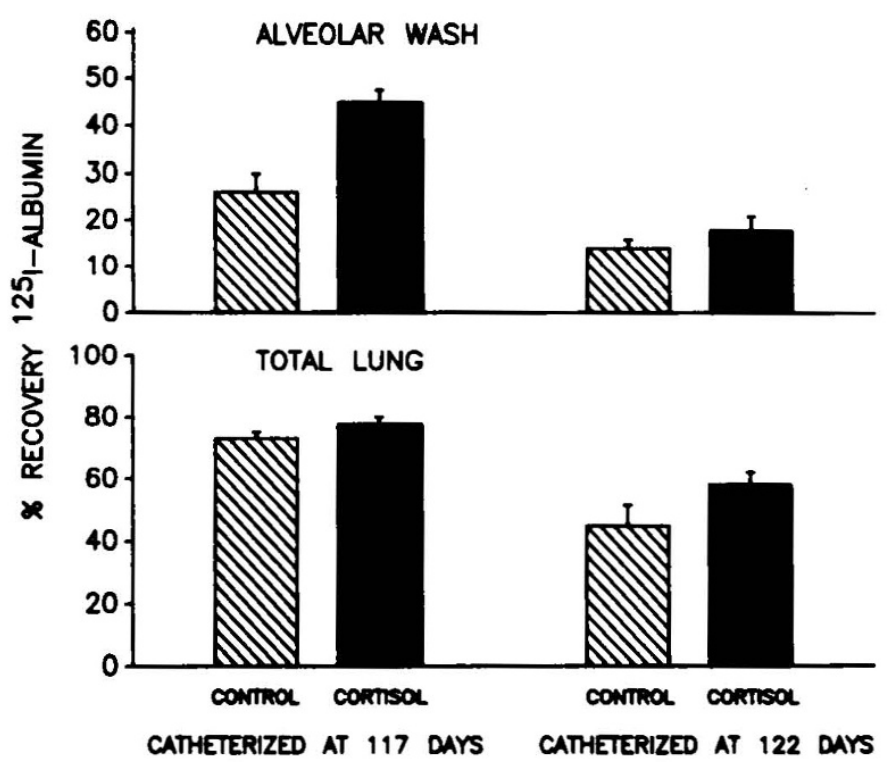

Fig. 3. Recovery of ${ }^{125} \mathrm{I}$-albumin mixed with fetal lung fluid at delivery in alveolar washes and total lung (alveolar wash plus lung tissue). The recovery in alveolar wash in the 117 -d cortisol animals was higher than that in the 122-d control group $(p<0.05)$. Total lung recoveries of ${ }^{125} \mathrm{I}$ albumin decreased as follows: $117-\mathrm{d}$ cortisol $>117-\mathrm{d}$ control $>122-\mathrm{d}$ cortisol $>122$-d control $(p<0.05)$. Animals numbers for each group are given in Table 1 .

the degree of prematurity at this gestation as reported by Liggins, Schellenberg, and their associates $(7,8)$. In an initial report, fetal catheters were placed at $124 \mathrm{~d}$ gestation, and infusions of various hormones, including cortisol, were begun within $24 \mathrm{~h}$ (7). The animals were studied at $128 \mathrm{~d}$ and surfactant pool measurements and pressure volumes curves were performed on unventilated fetal lungs. Cortisol, alone as well as in conjunction with other hormones, was found to have a beneficial effect on maximum lung volumes. We recently also reported large maturational effects of a 60-h cortisol infusion in lambs studied at 128-d gestational age (4). Subsequently, the Liggins group evaluated the effects of cortisol and TSH-releasing hormone (8). For these experiments, catheters were placed at $117 \mathrm{~d}$ and the infusions were begun $7 \mathrm{~d}$ later. With this design, cortisol alone had no effects on the fetal lungs. Taeusch et al. (19) also had reported inconsistent responses of fetal sheep to corticosteroids before 130 d gestational age.

These contradictory findings of the effect of fetal cortisol exposure led to the hypothesis of the present study. On the basis of the experiences of Liggins' group $(7,8)$, we theorized that surgical intervention alters the fetal endocrine milieu, making the animals receptive to hormonal induction of lung maturation. If hormonal treatment were initiated while the fetal state remained altered by the surgery, the fetus would respond to cortisol with accelerated pulmonary maturation. This receptive period might be short lived, given the lack of a corticosteroid response when therapy was initiated remote from the time of surgery (8). Fetal lung maturation normally is suppressed in favor of growth at early gestations. Once removed from the in utero environment, the fetal lung in explant cultures undergoes maturational changes that do not normally occur until much later in vivo. For example, explants of human fetal lung obtained at $15 \mathrm{wk}$ will rapidly differentiate and begin surfactant synthesis in the absence of hormonal stimulation (20). These changes can be accelerated by cortisol and/or other hormones, indicating that the tissue is receptive to hormone-induced maturation (21).

Our results do not support this hypothesis. In the animals in which surgery was performed $\geq 7 \mathrm{~d}$ before treatment, large corticosteroid-mediated effects on postnatal pulmonary function were noted. Cortisol treatment improved compliance by $50 \%$,
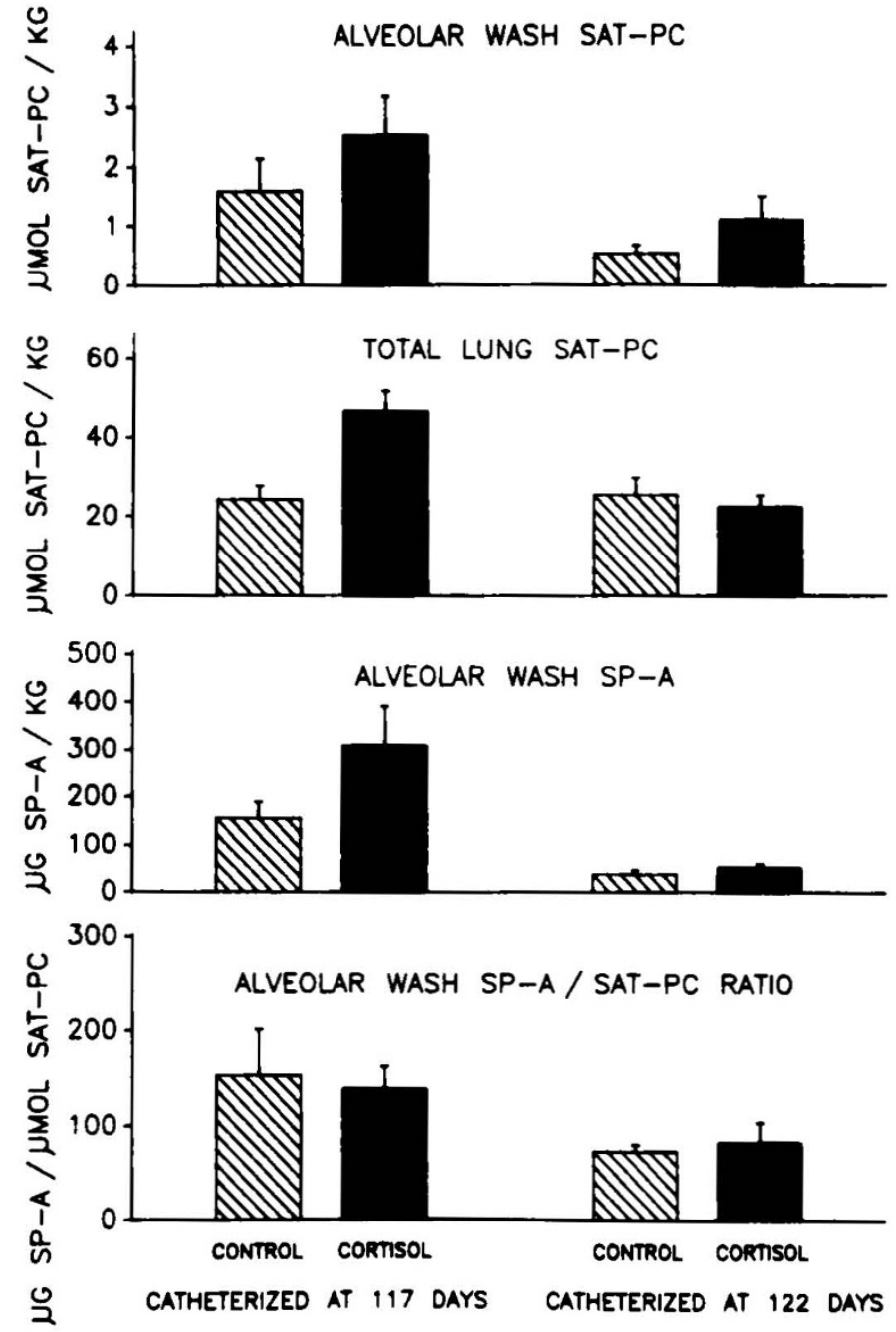

Fig. 4. Measurements of Sat PC and SP-A. Alveolar wash Sat PC recoveries were not different across the four groups. In contrast, total lung Sat PC (alveolar wash plus lung tissue) was significantly increased in the 117-d cortisol group relative to all other groups $(p<0.01)$. Alveolar SP-A was increased in the 117-d cortisol group relative to that in the 122-d groups $(p<0.01)$. The differences in the SP-A/Sat PC ratios were not significant. Animal numbers for each group are given in Table 1.

gas exchange as estimated by the ventilatory efficiency index improved by $150 \%$, and ventilatory pressure requirements were decreased by $40 \%$. The permeability of the alveolar epithelium/ vascular endothelium decreased to the levels measured in term newborn lambs. Several other interesting observations were made. For almost every end point, both controls and cortisoltreated animals catheterized at $117 \mathrm{~d}$ were more mature than their respective counterparts in the 122-d groups. Surprisingly, the 117-d control animals generally exhibit a level of lung function comparable to the 122-d cortisol-treated animals. Therefore, the intervention alone was as effective as the cortisol infusion when catheterization occurred at $117 \mathrm{~d}$ gestation. We subsequently have found that a single fetal dose of corticosteroids will reliably improve postnatal lung function in fetal sheep unexposed to a surgical intervention (22).

The improvements in lung function resulting from the intervention or cortisol infusions were associated with modest quantitative increases in alveolar Sat PC pool sizes. Cortisol treatment of the $117 \mathrm{~d}$ animals increased the amount of Sat PC by $50 \%$. This increase was not statistically significant but could represent a type II error because of the small sample size. However, the alveolar surfactant pools were very low in all groups $(<2.5 \mu \mathrm{mol} /$ $\mathrm{kg}$ ) compared with the amount of alveolar Sat PC found in term 
newborn sheep (about $100 \mu \mathrm{mol} / \mathrm{kg}$ ) (23). In contrast to the alveolar pools, the amount of Sat PC in the total lung in the 117d cortisol animals was twice that of the other groups. Therefore, the combination of early intervention and cortisol had a striking effect on lung tissue Sat PC pools.

The qualitative changes in the SP-A in alveolar washes were similar to the changes for Sat PC. The combination of 117-d catheterization and cortisol increased SP-A levels, whereas cortisol after intervention at $122 \mathrm{~d}$ had no effect. SP-A message is known to be responsive to corticosteroids (24). A single fetal dose of betamethasone in preterm lambs $48 \mathrm{~h}$ before delivery at $128 \mathrm{~d}$ induced increases in SP-A message but did not change SP$A$ in alveolar washes (25). The response in SP-A levels to both the 117-d intervention and cortisol infusion is intriguing in light of our previous observation that SP-A did not increase after cortisol or TSH-releasing hormone infusion in fetal sheep, but it increased 5-fold when both hormones were given simultaneously (4). Increases in SP-A in response to cortisol treatment may require the synergistic effects of other mediators such as catecholamines or thyroid hormones. We did not measure hormone levels in these animals, so we do not know what factors distinguish the 117-d from the 122-d interventions.

The results of this study raise questions regarding the interactive roles that fetal intervention and corticosteroids may play in natural and accelerated lung maturation. Our observations indicate that not only does the earlier intervention cause maturational changes in and of itself, it also results in an augmented response to cortisol. Perhaps once the fetal milieu is altered the fetal lung undergoes a slow premature maturation that is more sensitive to hormone-induced acceleration.

\section{REFERENCES}

1. Liggins GC 1969 Premature delivery of foetal lambs infused with glucocorticoids. J Endocrinol 45:515-523

2. Ballard PL 1986 Hormones and Lung Maturation. Monographs on Endocrinology, Vol 28. Springer-Verlag. Heidelberg, Germany

3. Crowley P. Chalmers I, Keirse M 1990 The effects of corticosteroid administration before preterm delivery: an overview of the evidence from controlled trials. Br J Obstet Gynecol 97:11-25

4. Ikegami M, Polk D, Tabor B, Lewis J, Yamada T, Jobe A 1991 Corticosteroid and thyrotropin-releasing hormone effects on preterm sheep lung function. J Appl Physiol 70:2268-2278

5. Ikegami M, Jobe A, Seidner S, Yamada T 1989 Gestational effects of corticosteroids and surfactant in ventilated rabbits. Pediatr Res 25:32-37
6. Stein HM, Kotaro Y, Martinez A, Chappell BA, Buhl E. Blount L, Padbury JF 1993 Effects of corticosteroids in preterm sheep on adaptation and sympathoadrenal mechanisms at birth. Am J Physiol 264:E763-E769

7. Schellenberg JC, Liggins GC, Manzai M. Kitterman JA, Lee CH 1988 Synergistic effects on lung maturation in fetal sheep. J Appl Physiol 65:94-100

8. Liggins GC. Schellenberg J, Manzai M, Kitterman JA, Lee CH 1988 Synergism of cortisol and thyrotropin-releasing hormone in lung maturation in fetal sheep. J Appl Physiol 65:1880-1884

9. Kotas RV, Avery ME 1971 Accelerated appearance of pulmonary surfactant in the fetal rabbit. J Appl Physiol 30:358-361

10. Mescher EJ, Platzker AC, Ballard PL, Kitterman JA, Clements JA, Tooley WH 1975 Ontogeny of tracheal fluid, pulmonary surfactant, and plasma corticoids in the fetal lamb. J Appl Physiol 39:1017-1021

11. Warburton D, Parton L, Buckley S, Cosico L. Enns G, Saluna T 1988 Combined effects of corticosteroid, thyroid hormones, and $\beta$-receptor binding in fetal lamb lung. Pediatr Res 24:166-170

12. Jobe A, Jacobs H, Ikegami M, Berry D 1985 Lung protein leaks in ventilated lambs: effects of gestational age. J Appl Physiol 58:1246-1251

13. Ikegami M. Jobe A. Yamada T, Priestley A. Ruffini L. Rider E, Seidner S 1989 Surfactant metabolism in surfactant treated preterm ventilated lambs. J Appl Physiol 67:429-437

14. Bligh EG, Dyer WJ 1959 A rapid method of total lipid extraction and purification. Can J Biochem Physiol 37:911-917

15. Mason RJ, Nellenbogen J. Clements JA 1976 Isolation of disaturated phosphatidyicholine with osmium tetroxide. J Lipid Res 17:281-284

16. Bartlett GR 1959 Phosphorous assay in column chromatography. J Biol Chem 234:466-468

17. Notter RH, Egan EA, Kwong MS, Holm BA. Shapiro DL 1985 Lung surfactant replacement in premature lambs with extracted lipid from bovine lung lavage: effects of dose, dispersion technique and gestational age. Pediatr Res 19:569-577

18. Jobe A, Ikegami M, Glatz T, Yoshida Y. Diakomanolis E, Padbury J 1981 The duration of characteristics of treatment of premature lambs with natural surfactant. J Clin Invest 67:370-375

19. Taeusch Jr HW, Avery ME, Sugg J 1972 Premature delivery without accelerated lung development in fetal lambs treated with long-acting methylpred. nisolone. Biol Neonate 20:85-92

20. Snyder JM, Johnston JM, Mendelson CR 198 I Differentiation of type II cells of human fetal lung in vitro. Cell Tissue Res 220:17-25

21. Gonzales LW, Ballard PL, Ertsey R, Williams MC 1986 Glucocorticoids and thyroid hormones stimulate biochemical and morphological differentiation of human fetal lung in organ culture. J Clin Endocrinol Metab 86:678-691

22. Jobe A, Polk D, Ikegami M, Newnham J, Sly P, Kohen R, Kelly R 1993 Lung responses to ultrasound-guided fetal treatments with corticosteroids in preterm lambs. J Appl Physiol 75:2099-2105

23. Rider E, Jobe A, Yamada T, Ikegami M, Seidner S, Ruffini L 1989 Surfactan metabolism during hyperventilation of newborn lambs with atrial right to left shunts. Pediatr Res 25:83-88

24. Schellhase DE, Shannon JM 1991 Effects of maternal dexamethasone on expression of SP-A, SP-B, and SP-C in the fetal rat lung. Am J Respir Cell Mol Biol 4:304-312

25. Austin SD, Polk D, Jobe A. Ikegami M 1993 Dose response of fetal betamethasone therapy for surfactant protein mRNA expression in preterm lambs. Pediatr Res 33:316A(abstr) 